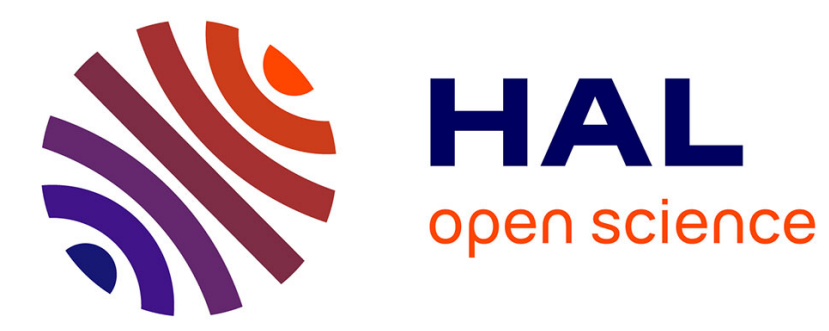

\title{
Circulations mathématiques et congruences dans les périodiques de la première moitié du XIX e siècle *
}

Jenny Boucard, Norbert Verdier

\section{To cite this version:}

Jenny Boucard, Norbert Verdier. Circulations mathématiques et congruences dans les périodiques de la première moitié du XIX e siècle * Philosophia Scientiae, 2015, Circulations et échanges mathématiques, 19 (2), pp.57-78. 10.4000/philosophiascientiae.1107 . halshs-01351695v2

\section{HAL Id: halshs-01351695 \\ https://shs.hal.science/halshs-01351695v2}

Submitted on 14 Apr 2021

HAL is a multi-disciplinary open access archive for the deposit and dissemination of scientific research documents, whether they are published or not. The documents may come from teaching and research institutions in France or abroad, or from public or private research centers.
L'archive ouverte pluridisciplinaire HAL, est destinée au dépôt et à la diffusion de documents scientifiques de niveau recherche, publiés ou non, émanant des établissements d'enseignement et de recherche français ou étrangers, des laboratoires publics ou privés. 
Philosophia

Scientie

\section{Philosophia Scientiæ}

Travaux d'histoire et de philosophie des sciences

$19-2$ | 2015

Circulations et échanges mathématiques

\section{Circulations mathématiques et congruences dans les périodiques de la première moitié du $\mathrm{XIX}^{\mathrm{e}}$ siècle}

Jenny Boucard et Norbert Verdier

\section{(2) OpenEdition}

Journals

Édition électronique

URL : http://journals.openedition.org/philosophiascientiae/1107

DOI : 10.4000/philosophiascientiae.1107

ISSN : 1775-4283

Éditeur

Éditions Kimé

Édition imprimée

Date de publication : 25 mai 2015

Pagination : $57-78$

ISSN : 1281-2463

Ce document vous est offert par Université de Nantes

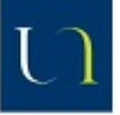

UNIVERSITÉ DE NANTES

Référence électronique

Jenny Boucard et Norbert Verdier, " Circulations mathématiques et congruences dans les périodiques de la première moitié du XIX siècle », Philosophia Scientiæ [En ligne], 19-2 | 2015, mis en ligne le 09 juin 2015, consulté le 09 avril 2021. URL : http://journals.openedition.org/philosophiascientiae/1107 ;

DOI : https://doi.org/10.4000/philosophiascientiae.1107 


\title{
Circulations mathématiques et congruences dans les périodiques de la première moitié du $\mathrm{XIX}^{\mathrm{e}}$ siècle*
}

\author{
Jenny Boucard \\ Centre François Viète, Université de Nantes (France) \\ Norbert Verdier \\ IUT Cachan \& GHDSO, Université Paris-Sud (France)
}

Résumé : Avec l'essor des journaux spécialisés, le paysage éditorial mathématique évolue considérablement pendant la première moitié du XIX ${ }^{e}$ siècle. Parallèlement, la publication des Disquisitiones arithmeticae de Gauss en 1801, avec son introduction de la notion de congruence, marque l'histoire de la théorie des nombres. Cet article propose une analyse de la double évolution du paysage éditorial et des congruences dans la première moitié du XIX siècle, en se concentrant sur les circulations mathématiques. Après avoir identifié le corpus des textes par lesquels la notion de congruence circule, nous en dressons un portrait général avant de nous focaliser sur des cas d'étude particulièrement significatifs. Congruences et périodiques sont ainsi envisagés, tour à tour, comme des objets et des moyens d'étude.

Abstract: With the rise of specialized journals, the landscape of mathematical publishing changed profoundly over the first half of the nineteenth century. Meanwhile, the publication of the Disquisitiones arithmeticae by Gauss in 1801, with its introduction of the concept of congruence, marks the history of number theory. This article analyzes the dual evolution of publishing landscape and congruences in the first half of the nineteenth century, focusing on mathematics circulations. Having identified the corpus of texts by which the notion of congruence circulates, we draw a general picture of it before focusing on case studies of particular significance. Congruences and periodicals are considered here as well, in turn, as objects and means of study.

\footnotetext{
*Nous remercions pour leurs commentaires stimulants et variés les deux rapporteurs, ainsi qu'Hélène Gispert et Pierre Teissier.
} 


\section{Introduction}

Au tournant du dix-neuvième siècle, pour publier des mathématiques, un savant dispose d'une offre éditoriale composée de périodiques mus par différents modèles dont voici quelques exemples : de type spécialisé comme les Archiv für reine und angewandte Mathematik de Carl Friedrich Hindenburg [Bullynck 2013], de type encyclopédiste comme le Magasin encyclopédique [Bret \& Chappey 2012] ou de type institutionnel, car rattaché à une institution académique ou d'enseignement (Journal de l'École polytechnique [Lamy 1995] ou Magazin für die Bergbaukunde à l'Académie des mines de Freiberg [Morel 2013, 189 et suiv.]). L'essor des journaux spécialisés s'intensifie dans le premier $\mathrm{XIX}^{\mathrm{e}}$ siècle [Verdier 2009b]. Ainsi, en 1810 est lancé le premier journal français exclusivement réservé aux mathématiques : les Annales de mathématiques pures et appliquées dites Annales de Gergonne du nom de son principal fondateur [Gérini 2014]. Ensuite, en 1825 et 1826, une première poussée éditoriale s'installe à Gand puis à Bruxelles avec la Correspondance mathématique et physique en 1825 par J. G. Garnier \& A. Quételet, à Vienne avec le Zeitschrift für Physik und Mathematik par A. Baumgartner \& A. Ettinghausen et à Berlin avec la création du Journal für die reine und angewandte Mathematik par A. L. Crelle, en 1826. À l'exception du Journal de Crelle qui existe encore, tous les autres ont cessé de paraître au cours des années 1830. Entre 1835 et 1837 sorte de seconde poussée éditoriale -, la presse périodique voit les lancements presque simultanés à Paris des Comptes rendus hebdomadaires de l'Académie des sciences, du Journal de mathématiques pures et appliquées par J. Liouville, puis la création en 1837 du Cambridge Mathematical Journal à Cambridge par R. L. Ellis \& D. F. Gregory. Quelques années plus tard, l'offre éditoriale s'étend à l'enseignement des mathématiques avec les Archiv der Mathematik und Physik par J. A. Grünert en Allemagne ${ }^{1}$ en 1841 et les Nouvelles annales de mathématiques en 1842, à Paris par C. Gérono \& O. Terquem.

Parallèlement à l'élargissement et à la diversification de l'offre éditoriale, le statut de la théorie des nombres, domaine marginal au début du XIX ${ }^{\mathrm{e}}$ siècle, évolue sensiblement tout au long du siècle. De nombreuses histoires de la théorie des nombres, publiées au $\mathrm{xx}^{\mathrm{e}}$ siècle, proposent un récit de cette évolution centrée sur quelques auteurs allemands - C. F. Gauss, C. G. J. Jacobi, J. P. G. Lejeune Dirichlet, E. E. Kummer, G. Eisenstein - et leurs travaux sur les résidus, lois de réciprocité et nombres idéaux interprétés comme des intermédiaires menant à la théorie moderne des nombres algébriques à la fin du $\mathrm{XIX}^{\mathrm{e}}$ siècle. Des travaux récents montrent les limites de cette lecture rétrospective, notamment à travers l'analyse de la réception - complexe et variée -

1. Dans la suite de l'article, nous désignons par Allemagne (respectivement Italie) le territoire géographique constitué de l'ensemble des États allemands (respectivement italiens) avant de devenir une entité politique, dans le second dix-neuvième siècle. 
des Disquisitiones arithmeticae de Gauss ${ }^{2}$, ouvrage publié en 1801 et considéré comme fondamental dans l'histoire de la théorie des nombres [Goldstein, Schappacher et al. 2007]. Les périodiques, qui deviennent alors le vecteur de circulation quasi exclusif des travaux arithmétiques pour les auteurs susmentionnés et pour les autres constituent un facteur matériel de cette transformation des pratiques arithmétiques. Un des objectifs de cet article est de comprendre les effets de la reconfiguration du paysage éditorial du premier XIX ${ }^{\mathrm{e}}$ siècle sur cette branche mathématique.

Nous avons choisi d'analyser cette double évolution du paysage éditorial et de la théorie des nombres dans le premier XIX ${ }^{\mathrm{e}}$ siècle à travers la notion mathématique de congruence, introduite par Gauss dans le premier article de son livre : deux nombres entiers $a$ et $b$ sont dits congrus modulo un nombre entier $n$ lorsque leur différence $a-b$ est divisible par $n$, c'est-à-dire qu'ils admettent le même reste après division euclidienne par $n$. Gauss introduit également la notation $: a \equiv b(\bmod n)$, soulignant ainsi l'analogie entre égalité et congruence.

Une analyse focalisée sur les congruences permet d'une part de saisir différentes formes d'appropriation de ce nouvel objet dans différents réseaux d'auteurs. Cette notion arithmétique peut d'autre part être envisagée comme un des marqueurs permettant d'analyser de manière systématique des aspects de l'évolution de la théorie des nombres, dont la définition est alors loin d'être stabilisée $^{3}$. La relative marginalité des congruences sur la période permet de plus d'envisager une étude s'appuyant sur un ensemble large de périodiques tout en obtenant un corpus de taille raisonnable. Les périodiques sont ici envisagés à la fois comme objet et moyen d'étude [Gispert 1999]. Moyens d'étude des congruences, ils sont envisagés comme des « lieux d'observation privilégiés pour étudier les transformations des savoirs et les modalités de diffusion » [Bret \& Chappey 2012] dans le cas des congruences. Réciproquement, se concentrer sur les congruences permet d'une part de préciser des modifications connues $\mathrm{du}$ paysage éditorial pour le premier $\mathrm{XIX}^{\mathrm{e}}$ siècle et d'autre part d'analyser concrètement des mécanismes de circulation des savoirs et des stratégies d'acteurs face à l'offre éditoriale. Les journaux deviennent alors objets d'étude et les congruences acquièrent à leur tour le double statut de sujet d'étude et de sonde historiographique.

2. Gauss y présente la théorie des nombres comme « cette partie des Mathématiques où l'on considère particulièrement les nombres entiers » [Gauss 1801, xi], introduit des concepts innovants, et propose une première exposition cohérente de nombreux résultats arithmétiques.

3. Par exemple, les définitions données par les acteurs ne concordent pas : lorsque Gauss définit la théorie des nombres comme la science des nombres entiers, des géomètres comme A.-M. Legendre ou P. Barlow confondent théorie des nombres et analyse diophantienne [Goldstein \& Schappacher 2007, 22-23]. De plus, les classifications proposées dans les journaux évoluent régulièrement, même lorsque l'on s'appuie sur des classifications proposées a posteriori dans les journaux de recension, par exemple [Goldstein 1999]. 
La première partie de cet article dresse un panorama général des congruences dans les périodiques dans le premier XIX ${ }^{\mathrm{e}}$ siècle. Les trois suivantes sont centrées sur des cas illustrant particulièrement notre propos : la deuxième traite du Bulletin de Férussac, ressource générale pour les congruences et marqueur de certaines tendances arithmétiques; la troisième montre la politique menée en faveur des congruences par Terquem, le principal rédacteur des Nouvelles annales de mathématiques; la dernière partie explore à travers deux mémoires les mécanismes de circulation mathématique.

\section{Les congruences de 1801 à 1850 : méthodologie et panorama général}

\subsection{Constitution d'un corpus de travail sur les congruences et premières données quantitatives}

Pour construire notre corpus [Boucard 2011a], nous n'avons pas défini un ensemble de périodiques à dépouiller pour éviter l'écueil de délimiter a priori les sources susceptibles de contenir des textes en lien avec les congruences. Nous avons donc dans un premier temps utilisé des synthèses postérieures sur la théorie des nombres comme [Dickson 1919-1923] pour obtenir un premier relevé de textes en lien avec les congruences. Nous avons ensuite procédé à un dépouillement des périodiques apparaissant dans ce premier relevé ${ }^{4}$. Dans chacun des cas, nous avons systématiquement recherché les différentes occurrences de mots et symboles clés renvoyant aux congruences et à la notion voisine de résidu ${ }^{5}$. En effet, le symbole de Gauss est loin d'être automatiquement utilisé au XIX ${ }^{\mathrm{e}}$ siècle. Legendre introduit par exemple la notation $M p$ pour désigner un multiple de $p$, qui est ensuite reprise par Binet ou Crelle par exemple. Pour chaque texte obtenu, nous avons appliqué une double grille de lecture, interrogeant les pratiques mathématiques en jeu, les références explicitées par les auteurs, intégrées dans notre corpus si les congruences y apparaissaient sous une forme ou une autre.

Notre corpus ${ }^{6}$ est constitué de 254 textes dont 27 seulement ne sont pas issus de périodiques : ouvrages, extraits d'encyclopédies ou mémoires parus

4. Cela s'avère nécessaire en raison notamment de la structure de l'ouvrage de Dickson qui exclut ainsi des travaux importants pour notre propos, comme nombre d'écrits de Kummer par exemple [Boucard 2011b, 22-27].

5. Comme Gauss le souligne, il existe un lien fort entre congruence et résidus, ces derniers étant déjà étudiés par Leonhard Euler (1707-1783) dans la seconde moitié du XVIII ${ }^{\mathrm{e}}$ siècle [Boucard 2011a, 124-190].

6. Les annexes insérées à la fin de cet article indiquent le nombre d'articles publiés par décennies pour chaque type de support imprimé (A) et pour chaque auteur (B). Nous avons également comptabilisé les différentes rééditions et traductions dont le rôle doit être pris en compte pour mieux saisir la circulation des contenus. 
indépendamment. Les autres écrits de notre corpus sont répartis dans une trentaine de périodiques, institutionnels ou privés (c'est-à-dire à but commercial), et sont de formes très diverses, allant de notes de quelques lignes à des mémoires de plusieurs centaines de pages. L'analyse quantitative du corpus confirme bien la double évolution du paysage éditorial et de la théorie des nombres sur le premier XIX ${ }^{\mathrm{e}}$ siècle : entre 1801 et 1810 , dix textes autour des congruences paraissent (dont six livres et deux articles dans des périodiques institutionnels), tandis qu'entre 1841 et 1850, 121 textes sont publiés, dont 36 dans des périodiques institutionnels et 80 dans des journaux mathématiques ou savants privés.

À partir de sa création en 1826, le Journal de Crelle est le support qui contient le plus de textes en lien avec les congruences (77 contre 31 dans les Comptes rendus de l'Académie des sciences de Paris), dont ceux considérés comme fondamentaux dans l'histoire de la théorie des nombres. Cela reflète également alors la situation particulièrement favorable de la théorie des nombres en Prusse, notamment grâce à la politique menée en faveur des jeunes arithméticiens prometteurs par A. von Humboldt et Gauss [Pieper 2007]. À la suite de ce dernier, Jacobi, Dirichlet, Kummer, Eisenstein et T. Schönemann travaillent sur les résidus, les lois de réciprocité et les nombres idéaux. Leurs écrits forment le cœur de nombreux récits historiques, et le fait que les références qui y sont mentionnées tendent à se refermer sur elles-mêmes ${ }^{7}$ permet de comprendre qu'une histoire construite à partir d'elles tende à occulter un nombre significatif d'acteurs et de travaux. En effet, sur les 56 auteurs apparaissant dans notre corpus, 18 sont allemands, publiant 122 textes et 22 sont français $^{8}$, produisant 111 textes. Le décalage entre l'importance quantitative des auteurs français et de leurs publications et la position marginale de ces derniers dans l'histoire de la théorie des nombres nous a conduits à concentrer notre analyse sur cet ensemble de travaux.

\subsection{Panorama des congruences à travers les journaux français}

Dans le premier quart de siècle, les auteurs - Legendre, S. F. Lacroix, Cauchy, L. Poinsot - sont académiciens et/ou polytechniciens et usent des périodiques associés à ces institutions pour diffuser leurs recherches arithmétiques. Ensuite, mis à part le Journal de Crelle, c'est un journal encyclopédique, le Bulletin des sciences mathématiques, astronomiques, physiques et chimiques mis en place par le baron de Férussac en 1823, qui héberge jusqu'à sa faillite au début des années trente le plus grand nombre de mémoires inédits (5) en lien avec les congruences et qui apparaît de plus comme une ressource générale pour les

7. Parmi les contemporains, et en dehors des auteurs allemands, seul L. A. Cauchy est cité à deux reprises après 1835 .

8. G. Libri est ici considéré comme français car il obtient la nationalité française au début des années 1830 [Maccioni Ruju \& Mostert 1995]. 
congruences, dans un sens précisé ultérieurement. Legendre joue un rôle non négligeable dans la diffusion des résultats d'auteurs exclus des institutions pour des raisons d'âge (Jacobi) ou de sexe (S. Germain) via des résultats de ces deux auteurs qu'il insère dans ses mémoires de théorie des nombres suite à des échanges épistolaires réguliers [Legendre 1827, 1830]. Signalons également quelques initiatives individuelles en termes de publications, comme la parution régulière des Exercices de mathématiques de Cauchy de 1826 à 1830 dont il est l'unique auteur, sous l'égide des éditeurs de Bure, membres de sa belle-famille [Belhoste 1985, 93] ou encore des mémoires de Libri et Dirichlet publiés à leurs frais.

Pour ce premier tiers de siècle, les discussions sur les congruences définissent donc un paysage éditorial spécifique, où plusieurs périodiques sont absents. C'est par exemple le cas des Annales de Gergonne $^{9}$, bien que J. D. Gergonne soit familier des recherches de Gauss ${ }^{10}$ : cela répond néanmoins aux objectifs des Annales, principalement destinées à l'enseignement et aux enseignants. En effet, les congruences sont absentes des différents programmes d'enseignement français [Belhoste 1995]. Les stratégies personnelles mises en œuvre par des auteurs comme Jacobi, Dirichlet ou encore Libri soulignent leurs difficultés pour publier leurs recherches, difficultés d'autant plus importantes au cours des années 1830, au moment où, à l'exception du Journal de Crelle, les entreprises éditoriales évoquées précédemment cessent leurs activités pour diverses raisons.

À partir de 1835, les différents articles sont alors distribués dans le triangle constitué des Comptes rendus de l'Académie des sciences, du Journal de Liouville et des Nouvelles annales de mathématiques. Comme dans le cas plus général des mathématiques, cette fragmentation du paysage éditorial est combinée à l'absence d'une ressource générale pour les congruences. Une originalité pour la thématique des congruences réside dans le fait que pour chacun de ces trois périodiques, un acteur se distingue tout particulièrement : Cauchy pour les Comptes rendus de l'Académie (avec 22 notes sur 31), V. A. Lebesgue pour le Journal de Liouville ( 8 sur les 11 mémoires inédits) et Terquem pour les Nouvelles annales (7 sur 17).

Si la notion de congruence est assimilée de manière très hétérogène parmi les différents auteurs français, ils considèrent majoritairement les congruences dans un lien très fort avec les équations. Ainsi, des problématiques spécifiques en ressortent : des analogies entre équations et congruences binômes chez Poinsot, la considération d'équations indéterminées d'une forme donnée chez Cauchy, la détermination du nombre de solutions des congruences chez Lebesgue, une application des congruences à la théorie algébrique des équations chez Galois, ou encore des recherches sur le dernier théorème de Fermat

9. Seul Libri mentionne les congruences pour présenter une méthode permettant d'éviter leur usage [Libri 1825].

10. Il avait en effet entrepris une traduction des Disquisitiones arithmeticae avant de prendre connaissance de la traduction de A.-C.-M. Poullet-Delisle publiée en 1807 [Gergonne 1821, 337]. 
chez Legendre et Germain. Comme cette liste en témoigne, les pratiques sousjacentes sont très diverses et se situent dans des représentations disciplinaires multiples ${ }^{11}$. Lebesgue semble progressivement se singulariser par ses thématiques de recherche - résidus cubiques et biquadratiques par exemple -, qui sont proches de celles des auteurs allemands évoqués précédemment.

Notons qu'un même texte peut exister sous différentes formes au sein de cet espace éditorial, à travers les comptes rendus, rééditions ou traductions ${ }^{12}$, ce qui reflète plus généralement une pratique éditoriale courante. Des rééditions, traductions ou développements de mémoires parus dans les publications académiques (de Paris, de Berlin) sont en effet régulièrement insérés dans les journaux privés. Ainsi, dans son article de 1845, Poinsot propose en introduction un véritable manifeste en faveur de la théorie des nombres et son intégration dans l'enseignement, puis expose plusieurs démonstrations de théorèmes arithmétiques fondamentaux de la théorie des nombres [Poinsot 1845]. L'introduction est publiée dans les Comptes rendus en 1841, avant que le mémoire complet soit inséré dans le Journal de Liouville quatre ans plus tard. De même, sur les neuf notes de Cauchy publiées dans les Comptes rendus, deux sont reproduites dans le Journal de Liouville. Cette sélection semble d'ailleurs avoir été opérée pour donner à voir un échange d'arguments entre Cauchy et Lebesgue $^{13}$. Enfin, après 1835 , cinq articles déjà publiés dans le Journal de Crelle ou par l'Académie de Berlin sont insérés - directement ou après avoir été traduits - dans le Journal de Liouville et Terquem propose des adaptations de plusieurs textes de L. Euler, Dirichlet ou encore L. Kronecker dans les Nouvelles annales de mathématiques. Le procédé inverse - une réédition de texte dans le sens Paris-Berlin - ne se produit en revanche jamais dans le cas des congruences, bien que cela soit exceptionnellement le cas dans d'autres domaines mathématiques (analyse ou géométrie) comme des démonstrations d'un théorème sur l'isopérimétrie par J. Steiner en 1841-1842 [Verdier 2009a, 299-305].

11. Les travaux de Galois, Libri ou Cauchy peuvent être rattachés au champ de recherche analyse algébrique arithmétique défini dans [Goldstein \& Schappacher 2007], lorsque Poinsot propose une approche liant la théorie des nombres, l'algèbre et la géométrie, sous le nom de théorie de l'ordre [Boucard 2011b].

12. Trente textes du corpus sont de cette nature.

13. Ainsi, dans les séances de l'Académie des 6 et 13 avril 1840 [Cauchy 1840a,b], Cauchy cite [Lebesgue 1840a], paru dans le Journal de Liouville en février 1840. Lebesgue publie ensuite une Note sur un théorème de Cauchy [Lebesgue 1840b], dans laquelle il revient sur [Cauchy 1840a]. 


\section{Le Bulletin de Férussac : une ressource générale pour les congruences (1823-1831)}

Avec son Bulletin, Férussac met en place une publication de type universaliste développée autour d'une structure de sociabilité scientifique, centrée sur sa bibliothèque-salon, connue pour être un lieu de rencontres privilégié pour les différents collaborateurs [Verdier 2012]. À l'instar du salon tenu par Crelle pour animer son journal, le salon de Férussac permet notamment à de jeunes savants comme Dirichlet ou É. Galois d'emprunter des ouvrages mathématiques, d'en discuter et, au final, de partager des références mathématiques communes.

Dans son article, R. Taton affirme que le Bulletin de Férussac offre « une image fidèle [...] des différentes tendances qui se font jour dans les recherches » [Taton 1947]. Notre étude confirme ce propos et permet de préciser les pratiques des acteurs. Tout d'abord, parmi les quatre auteurs publiant des recherches inédites, Cauchy est académicien depuis 1816 et Libri vient de voir accepter un « Mémoire sur la théorie des nombres» pour publication dans les Mémoires des savants étrangers de l'Académie. Ils choisissent pourtant d'insérer dans le Bulletin certains de leurs travaux sous forme de notes succinctes ou d'articles d'une dizaine de pages, probablement pour pallier les lenteurs de l'institution. Le Bulletin permet également de faire connaître les travaux de Lebesgue et Galois ${ }^{14}$ qui sont présentés à l'Académie sans être acceptés pour publication.

À côté des articles inédits, le lectorat du Bulletin a également accès à des résumés de présentations à l'Académie, des annonces de parution d'ouvrages et des comptes rendus d'articles. Une comparaison entre le contenu du Bulletin et la liste des différentes publications en lien avec notre thématique pour la période 1824-1830 montre que seuls six textes ne sont pas répertoriés ${ }^{15}$. Par exemple, à chaque mémoire publié dans le Journal de Crelle correspond un compte rendu dans le Bulletin de Férussac rédigé par A. A. Cournot, qui s'est fortement impliqué dans le Bulletin dès 1824 [Cournot 2010, 17]. C'est dans ce sens que le Bulletin peut être qualifié de ressource générale : il propose l'actualité arithmétique dans son ensemble, ou presque, qu'elle soit initialement publiée en français ou non. La maîtrise de l'allemand par Cournot permet ainsi de rendre compte des textes publiés dans le Journal de Crelle dont le contenu est difficilement accessible pour nombre de géomètres français pour des raisons d'ordre linguistique [Cournot 2010, 129]. Ses comptes rendus constituent une référence pour certains auteurs, comme l'indique Lebesgue en 1839 :

14. Sur Lebesgue, voir [Abria \& Houël 1876]; sur Galois, voir [Ehrhardt 2011].

15. Ces différents textes sont issus de sources plus difficiles d'accès [Lebesgue 1829a,b,c] ou sont très courts [Verhulst 1827]. Le contenu des Exercices de Cauchy n'est également plus résumé après la $42^{\mathrm{e}}$ livraison (août 1829). 
Je ne connaissais pas le mémoire de M. Dirichlet quand j'ai trouvé la démonstration du théorème général (I) donné plus haut, mais j'avais lu ce qu'on en dit dans le Bulletin des sciences mathématiques de M. Férussac. [Lebesgue 1839, 59]

Les textes de Cournot renseignent de plus sur les thématiques qu'il considère comme décisives. Ainsi, dans un compte rendu sur une courte note de Jacobi sur les résidus cubiques [Jacobi 1827], Cournot commence par une courte introduction historique dans laquelle il rappelle, à la suite de Jacobi, que Gauss «promet depuis longtemps au public » [Cournot 1827a] de nouveaux résultats sur les résidus cubiques. Il souligne également la nécessité de développer une " théorie neuve et piquante», celle des racines imaginaires de congruences, en citant les travaux de Poinsot. Il est intéressant de constater que Cournot rapproche les travaux de Poinsot, Gauss et Jacobi. En effet, Galois publie en 1830 des recherches liées à la résolution algébrique des équations et dans lesquelles il introduit les racines imaginaires des congruences irréductibles modulo un nombre premier $p$ [Galois 1830]. L'historiographie tend à faire de Galois un auteur isolé sur cette thématique [Frei 2007] ; or, son travail s'inscrit en fait dans un ensemble de travaux dont les contenus sont signalés dans le Bulletin et ce compte rendu de Cournot suggère au contraire que la question des racines imaginaires de congruences est considérée comme intéressante dès les années 1820 .

\section{Les Nouvelles annales de mathéma- tiques ou la promotion de la théorie des nombres dans la presse intermédiaire (1842-1850)}

Si les Nouvelles annales de mathématiques, « journal des candidats aux Écoles polytechnique et normale », visent un public constitué d'enseignants et d'étudiants, la présence d'une quinzaine d'articles en lien avec les congruences publiés entre 1842 et 1850 offre un contraste avec la situation du début du siècle et les Annales de Gergonne. Ici, Terquem, un des deux fondateurs du périodique, utilise sa publication pour être une force de proposition afin d'intégrer dans l'enseignement des éléments de théorie des nombres. L'étude des textes souligne la volonté répétée de Terquem de «propager » [Midy 1845, 147] les congruences. Auteur de cinq articles dans lesquels il reprend des résultats élémentaires et des preuves de Gauss, Dirichlet, Euler ou encore Kronecker, il propose également des comptes rendus critiques des manuels d'arithmétique de Guilmin et Cirodde, dans lesquels il déplore l'absence de la théorie des congruences qui permettrait de simplifier l'exposé du contenu mathématique [Terquem 1842, 54]. En tant qu'éditeur, il intervient à plusieurs reprises dans 
les articles pour souligner l'intérêt de diffuser une théorie des nombres fondée sur les résidus et les congruences. Il introduit également la notation $\dot{p}$, désignant un multiple quelconque d'un nombre entier $p$, qui selon lui présente « un avantage pour le calculateur et aussi sous le rapport typographique » [Terquem 1844, 343]. Elle est systématiquement utilisée jusqu'en 1849, dans dix articles contre trois où la notation de Gauss apparaît entre 1850 et 1862. La première apparition du symbole $\equiv$ dans ce journal coïncide avec un changement d'éditeurs du journal : éditées les premières années par Carilian-Goeury, l'édition des Nouvelles annales est reprise en 1849 par Bachelier, qui est alors l'imprimeur par excellence pour les mathématiques [Verdier 2013a]. Néanmoins, la persistance de cette notation dans les années 1850 suggère qu'au-delà d'une possible limitation typographique, cette spécificité semble être une marque de fabrique des Nouvelles annales.

À côté de Terquem, sept auteurs publient un article chacun et E. Prouhet en publie quatre. C. Drot, É. Midy et Prouhet sont enseignants dans des collèges de province et Thibault à Paris, E. Catalan est répétiteur à l'École polytechnique, et S. Réalis est ingénieur civil. Lebesgue, professeur à l'université de Bordeaux depuis 1838, est le seul à publier sur les congruences dans d'autres supports que les Nouvelles annales. Comme Terquem, les auteurs proposent une présentation accessible aux débutants de résultats classiques de la théorie des résidus et des congruences. Catalan, Thibault et Midy traitent des fractions périodiques et l'analyse indéterminée du premier degré, qui figurent aux programmes d'enseignement [Catalan 1842], [Thibault 1843], [Midy 1845]. Les auteurs insistent régulièrement sur l'intérêt de reprendre des sujets connus à l'aide des congruences et soulignent à plusieurs reprises les objectifs pédagogiques de leur projet : ainsi, Prouhet clôt son article avec un exercice consistant à trouver des preuves de deux résultats énoncés par Euler [Prouhet 1846]. En l'absence de tout manuel de théorie des nombres en français, et même s'il est difficile de mesurer l'effet sur le lectorat, les Nouvelles annales constituent un lieu d'échanges privilégié sur cette thématique pour des enseignants et des étudiants parfois éloignés géographiquement ${ }^{16}$.

\section{Circulations arithmétiques entre Paris et Berlin}

\subsection{Un « Mémoire sur la théorie des nombres » de Libri (1824-1834)}

Le cas d'un travail de Libri sur la théorie des nombres témoigne bien des mécanismes de circulation mathématique. Présenté à l'Académie des sciences

16. Notons que ce ne sont pas les seuls textes témoignant d'un souhait de voir la théorie des nombres intégrer les programmes d'enseignement en France : c'est le cas de [Poinsot 1845] mentionné précédemment. 
de Paris entre 1824 et 1825 et accepté pour publication dans les Mémoires des savants étrangers, il n'y sera inséré qu'en 1838. Pour pallier les lenteurs académiques, coutumières aussi bien à Berlin qu'à Paris, Libri utilise un autre canal éditorial : il fait publier à ses frais son mémoire chez le libraire florentin L. Ciardetti [Libri 1829]. Mais, comme il le souligne lui-même, ce texte, ainsi que d'autres mémoires « sont restés inconnus à la librairie et au public » [Libri $1831,57]$. Trois années plus tard, une version très proche de son texte est publiée dans le Journal de Crelle, témoignage d'une proximité scientifique et humaine avérée entre Libri et Crelle [Libri 1832]. Ainsi, c'est Libri qui transmet directement à Crelle, lors de ses voyages à Berlin, certains mémoires de savants français comme Liouville, car, en cette période, les lenteurs postales entre Paris et Berlin sont relevées par plusieurs acteurs [Verdier 2009a, 38-43].

Crelle sollicite de plus l'imprimeur de son journal, C. Reimer, pour faire publier en 1835 un ensemble de dix mémoires de Libri, contenant toujours le « Mémoire sur la théorie des nombres ${ }^{17}$. Ce mémoire arithmétique de Libri est ainsi publié par quatre canaux éditoriaux différents en neuf ans et l'éditeur Crelle joue un rôle fondamental dans sa circulation outre-Rhin.

Dans ce texte, Libri s'appuie particulièrement sur les écrits de Gauss et Poinsot, mais propose une approche tout à fait originale de la théorie des congruences, qu'il fait dépendre de l'analyse. Comme Poinsot, il s'appuie sur des analogies entre équations et congruences, mais il produit des preuves fondées sur la considération d'un critère d'intégralité des nombres défini à partir des fonctions circulaires ${ }^{18}$. Or, il est fort probable que ce soit la publication dans le Journal de Crelle qui ait inauguré la circulation effective des idées de Libri. Ainsi, M. A. Stern publie ses recherches arithmétiques dans le Journal de Crelle à partir de 1830. C'est cependant en 1834 qu'il s'appuie explicitement sur les méthodes de Libri, héritées de Poinsot, pour proposer des preuves algébriques de théorèmes arithmétiques - comme la résolution de la congruence $x^{2} \equiv-3(\bmod p)-$, dont certains étaient déjà démontrés dans ses mémoires antérieurs, mais « par d'autres voies » [Stern 1834, 291].

Cet exemple montre comment un même contenu reste plus ou moins anonyme ou est réutilisé selon son support de publication. Il illustre deux mécanismes de circulation. D'une part, les recherches de Libri restent vraisemblablement dans l'anonymat tant qu'elles ne sont pas associées à un vecteur de circulation adéquat, à savoir le Journal de Crelle. D'autre part, l'article de Libri publié dans le Journal de Crelle semble être un véhicule pour des méthodes de Poinsot dont on ne retrouve de traces à Berlin auparavant, bien qu'elles aient été connues dans les réseaux parisiens.

17. C'est dans la préface de ce volume que Crelle annonce qu'il avait demandé à Libri « la permission de faire réimprimer et de publier par la voie du journal ces excellents morceaux » [Libri 1835, iii].

18. Pour traduire le fait qu'un nombre $x$ est entier, il considère l'équation $\sin x \pi=0$. 


\subsection{Un mémoire de Jacobi de Berlin à Paris (1837-1856)}

Arrêtons-nous pour finir sur un mémoire de Jacobi, qui est publié à trois reprises entre 1837 et 1856 . Ce texte de Jacobi, intitulé Über die Kreistheilung und ihre Anwendung auf die Zahlentheorie, contient des résultats fondamentaux sur les sommes de Gauss et de Jacobi, des applications à la loi de réciprocité cubique, aux formes quadratiques, et correspond en partie au contenu d'un cours que Jacobi donne à l'université de Königsberg pendant le semestre d'hiver 1836-1837 [Jacobi 2007]. Il est publié une première fois dans les comptes rendus de l'Académie de Berlin en 1837 [Jacobi 1837], puis est réédité dans le Journal de Crelle neuf ans plus tard [Jacobi 1846]. Il est finalement traduit en français par un jeune polytechnicien encouragé par Terquem, E. N. LaguerreWerly, et inséré dans les Nouvelles annales en 1856 [Jacobi 1856].

Contrairement à ce qui précède, nous n'avons retrouvé aucun commentaire sur les raisons qui ont poussé les acteurs en jeu à rééditer ce texte. Le texte de Jacobi est néanmoins connu assez rapidement par plusieurs auteurs, puisqu'il est explicitement cité par Cauchy, Dirichlet, Eisenstein, Kronecker, Lebesgue ou encore Schönemann avant 1846. Nous pouvons supposer que, dans le cas du Journal de Crelle comme dans celui des Nouvelles annales, les éditeurs respectifs ont choisi de reproduire ce texte non pas parce qu'il est tombé dans l'oubli comme dans le cas de Libri précédemment analysé, mais parce qu'il constitue à leurs yeux une référence arithmétique importante.

Le contenu de ces trois versions est le même, mais des ajouts sont progressivement intégrés par différents protagonistes. Ainsi, en 1846, Jacobi précise dans plusieurs notes quels travaux relatifs au même thème ont été publiés depuis 1837, et semble ainsi vouloir situer son travail par rapport aux très nombreux articles publiés dans le Journal de Crelle depuis. Il joint de plus des tables de décomposition de nombres premiers en sommes de carrés, calculées pour la plupart par des auditeurs de son cours, dont A. R. Zornow, régent du gymnase de Kneiphausen à Königsberg. Ce dernier a d'ailleurs collaboré avec Jacobi à plusieurs reprises : en effet, il publie des tables de décompositions en sommes de cubes en 1835 calculées dans le but de vérifier un théorème à la demande de Jacobi [Zornow 1835] et Jacobi se réfère dans un autre mémoire à d'autres tables de décompositions en sommes de carrés calculées par Zornow [Jacobi 1839].

Dans la traduction de 1856, aux premières notes de Jacobi et celles ajoutées en 1846 sont jointes des notes du traducteur Laguerre et de l'éditeur Terquem, qui complètent notamment les références de Jacobi en évoquant les travaux de Cauchy, Dirichlet, Lebesgue, Kummer, Libri ou encore J.-A. Serret. Les tables numériques ne sont en revanche pas reproduites, sans aucune explication. Ce cas se présente également dans le cas d'un compte rendu par Terquem de [Zornow 1835] inséré dans les Nouvelles annales en 1850, bien que Terquem qualifie la table de Zornow d' « ingénieuse ». Au-delà de l'absence des tables, la traduction de ce texte non élémentaire de Jacobi dans les Nouvelles 
annales atteste d'une volonté de Terquem de plaider en faveur de l'intégration de la théorie des nombres dans son journal en présentant en français des résultats alors classiques de la théorie des nombres et en les complétant par des références bibliographiques accessibles en France sachant que les textes de Dirichlet et Kummer cités ont déjà été publiés dans le Journal de Liouville.

\section{Conclusion}

L'exploration d'un ensemble de journaux contenant des mathématiques afin de constituer un corpus autour des congruences permet de mettre au jour des pratiques mathématiques variées : ainsi, à côté des articles publiés par des auteurs allemands dans le Journal de Crelle et les périodiques institutionnels berlinois et dont l'inter-citation est forte, nous avons notamment montré l'existence d'un autre ensemble de textes, dont les liens sont plus lâches et dont les auteurs évoluant en France proposent des recherches arithmétiques ancrées dans un rapprochement étroit entre équations et congruences. La prise en compte de tous les textes du corpus - y compris les comptes rendus, rééditions et traductions - met au jour différentes formes de circulations mathématiques. Par exemple, les comptes rendus de Cournot dans le Bulletin de Férussac donnent à voir les liens que ce dernier fait entre les recherches de Gauss, Jacobi et Poinsot. Les différentes traductions et reprises de textes de Jacobi et Dirichlet insérées dans le Journal de Liouville et les Nouvelles annales de mathématiques constituent un marqueur des thématiques arithmétiques estimées par les rédacteurs de ces périodiques. Le "Mémoire sur la théorie des nombres » de Libri publié en 1832 dans le Journal de Crelle semble être un vecteur pour des méthodes publiées par Poinsot une décennie plus tôt puisque celles-ci sont ensuite reprises par Stern en 1834.

Si la marginalité de la position de Lebesgue, comparée au nombre de ses publications arithmétiques, montre bien qu'une spécialisation dans le domaine de l'arithmétique est insuffisante pour assurer facilement une carrière académique en France, la volonté de plusieurs acteurs d'intégrer la théorie des nombres, et ce, particulièrement dans l'enseignement, est soulignée. C'est par exemple le cas de Poinsot ou de Terquem qui, en tant que rédacteur et auteur des Nouvelles annales, montre son souhait de diffuser la théorie des congruences, en introduisant une notation spécifique et en présentant des versions accessibles aux débutants de résultats démontrés antérieurement par Euler, Gauss, Dirichlet ou encore Cauchy.

Un sujet qui reste marginal tout au long de la période considérée, comme les congruences, permet de montrer sous une forme spécifique des caractéristiques du paysage éditorial pour la première moitié $\mathrm{du}$ XIX ${ }^{\mathrm{e}}$ siècle, comme sa fragmentation à la fin des années 1830 , les relations entre les périodiques institutionnels et privés, ou encore les rôles fondamentaux de certains acteurs comme Cournot, Crelle, Liouville ou Terquem. De plus, via notre objet arith- 
métique apparaît une cartographie propre, largement dominée par une intense pulsation entre Paris et Berlin avec leurs publications académiques et privées. Certains périodiques pourtant reconnus n'apparaissent pas : ainsi, avant 1835 et en dehors des périodiques institutionnels, seuls le Bulletin de Férussac et le Journal de Crelle contiennent plus d'un article en lien avec les congruences. De même, nous observons une quasi-absence d'articles parus dans les journaux mathématiques britanniques créés dans les années 1830. Même si les éditeurs respectifs des Annales de Gergonne et des Nouvelles annales de mathématiques sont familiers de la théorie des nombres de Gauss, la position de celle-ci dans leurs journaux respectifs est très différente. Notons que les congruences sont totalement absentes de la rubrique «Questions-Réponses » des Nouvelles annales, suggérant une plus grande concordance entre celle-ci et les programmes d'enseignement.

Cette analyse confirme que le clivage Comptes rendus/ Journal de Liouville/ recherche et Nouvelles annales/ enseignement n'est pas si clair : les premiers contiennent des articles présentant des résultats élémentaires de théorie des nombres [Binet 1841], [Poinsot 1845], quand Terquem fait traduire, avec un décalage dans le temps certes, une référence fondamentale de Jacobi sur la cyclotomie et les formes quadratiques dans son journal. Se focaliser sur un thème mathématique circonscrit permet également de suivre les stratégies mises en œuvre par les acteurs - auteurs et éditeurs notamment - et certains mécanismes de circulation des mathématiques. Nous l'avons vu dans les cas de Libri et Jacobi. La sélection de deux notes aux Comptes rendus de Cauchy parmi neuf pour reproduction dans le Journal de Liouville souligne également le poids éditorial de Lebesgue, véritable interlocuteur privilégié pour Liouville en matière de théorie des nombres.

Finalement, ces études de cas, consistant à étudier une sélection de textes et de supports focalisés sur une thématique donnée, sont à multiplier pour cerner avec plus d'acuité les diversités des acteurs (savants, institutions et publications impliqués) et des pratiques éditoriales afin d'établir des cartographies donnant à voir le paysage éditorial sous différents angles de vue.

\section{Bibliographie}

AbriA, Joseph-Benoît \& Hoü̈L, Jules [1876], Notice sur la vie et les travaux de Victor-Amédée Lebesgue, Bulletino di bibliografia e di storia delle scienze matematiche e fisiche, 9, 554-582.

Belhoste, Bruno [1985], Cauchy, un mathématicien légitimiste au XIX ${ }^{e}$ siècle, Paris : Belin.

[1995], Les Sciences dans l'enseignement secondaire français. Textes officiels, 1789-1914, Paris : INRP/Économica. 
Binet, Jacques Philippe Marie [1841], Note sur une nouvelle méthode pour trouver le plus grand commun diviseur des nombres entiers, ou des polynômes algébriques, et sur l'application de cette méthode aux congruences du premier degré, $C R A S^{19}, 13,349-353$.

BoucARD, Jenny [2011a], Un « rapprochement curieux de l'algèbre et de la théorie des nombres ": études sur l'utilisation des congruences de 1801 à 1850, Thèse de doctorat, université Paris 6.

— [2011b], Louis Poinsot et la théorie de l'ordre : un chaînon manquant entre Gauss et Galois?, Revue d'histoire des mathématiques, 17(1), 41-138.

Bret, Patrice \& Chappey, Jean-Luc [2012], Spécialisation vs encyclopédisme?, La Révolution française. Cahiers de l'Institut d'histoire de la Révolution française, 2, en ligne, URL http://lrf.revues.org/515.

BulLYNCK, Maarten [2013], Stages towards a German mathematical journal (1750-1800), Archives Internationales d'Histoire des Sciences, 63(170-171), $237-251$.

Catalan, Eugène-Charles [1842], Sur les fractions décimales périodiques, NAM, I(1), 457-470.

Cauchy, Augustin-Louis [1840a], Méthode simple et nouvelle pour la détermination complète des sommes alternées, formées avec les racines primitives des équations binômes, CRAS, 10, 560-572.

_ [1840b], Sur la sommation de certaines puissances d'une racine primitive d'une équation binôme, et en particulier, des puissances qui offrent pour exposants les résidus cubiques inférieurs au module donné, $C R A S, 10,594-$ 606.

Cournot, Antoine-Augustin [1827a], Des résidus cubiques; par le dr. jacobi, Bulletin des sciences mathématiques, astronomiques, physiques et chimiques, 8, 302 .

[2010], Euvres complètes T. XI : Écrits de jeunesse et pièces diverses, Paris; Besançon : Vrin ; Presses universitaires de Franche-Comté, édité par B. Bru et T. Martin.

Dickson, Leonard Eugene [1919-1923], History of the Theory of Numbers, Washington : Carnegie Institute of Washington, 3 vol.

Ehrhardt, Caroline [2011], Évariste Galois. La fabrication d'une icône mathématique, Paris : Éditions EHESS.

19. Nous désignons les principaux périodiques par les sigles indiqués dans l'annexe A. 
FREI, Günther [2007], The unpublished section eight? : On the way to function fields over a finite field, dans The Shaping of Arithmetic after C.F. Gauss's Disquisitiones Arithmeticae, édité par C. Goldstein, N. Schappacher \& J. Schwermer, Berlin : Springer, 159-198.

Galois, Évariste [1830], Sur la théorie des nombres, Bull. Fér., 13, 428-435.

Gauss, Carl Friedrich [1801], Disquisitiones Arithmeticae, Leipzig : Fleischer, cité d'après la traduction française par A. C. M. Poullet-Delisle : Recherches arithmétiques, Paris : Courcier, 1807.

Gergonne, Joseph Diaz [1821], Philosophie mathématique. Dissertation sur la langue des sciences en général, et en particulier sur la langue des mathématiques, AMPA, 12, 322-359.

GÉRInI, Christian [2014], Les Annales de mathématiques pures et appliquées de Gergonne et l'émergence des journaux de mathématiques dans l'Europe $\mathrm{du} \mathrm{XIX}^{\mathrm{e}}$ siècle, dans L'Émergence de la presse mathématique en Europe au $\mathrm{XIX}^{e}$ siècle. Formes éditoriales et études de cas (France, Espagne, Italie $\mathcal{E}$ Portugal), édité par C. Gérini \& N. Verdier, Londres : College Publications, $5-36$.

Gispert, Hélène [1999], Champs conceptuels et milieux mathématiques : objets et moyens d'études, méthodes quantitatives en histoire des mathématiques, Acta historiae rerum naturalium necnon technicarum, III(28), 167185.

Goldstein, Catherine [1999], Sur la question des méthodes quantitatives en histoire des mathématiques : le cas de la théorie des nombres en France (1870-1914), Acta historiae rerum naturalium necnon technicarum, 28, 187214.

Goldstein, Catherine \& Schappacher, Norbert [2007], A book in search of a discipline (1801-1860), dans The Shaping of Arithmetic after C. F. Gauss's Disquisitiones Arithmeticae, édité par C. Goldstein, N. Schappacher \& J. Schwermer, Berlin : Springer, 3-65.

Goldstein, Catherine, Schappacher, Norbert, \& Schwermer, Joachim (éd.) [2007], The Shaping of Arithmetic after C. F. Gauss's Disquisitiones Arithmeticae, Berlin : Springer.

JACOBI, Carl Gustav Jacob [1827], De residuis cubicis commentatio numerosa, Journal für die angewandte Mathematik, 2, 66-69.

[1837], Über die Kreistheilung und ihre Anwendung auf die Zahlentheorie, Monatsbericht der Akademie der Wissenschaften zu Berlin, 127-136. 
[1839], Über die complexen Primzahlen, welche in der Theorie der Reste der 5ten, 8ten und 12ten Potenzen zu betrachten sind, J. Crelle, 19, 314318.

— [1846], Über die Kreistheilung und ihre Anwendung auf die Zahlentheorie, J. Crelle, 30, 166-182.

[1856], Sur la division du cercle et son application à la théorie des nombres, NAM, I(15), 337-352.

[2007], Vorlesungen über Zahlentheorie-Wintersemester 1836/37, Königsberg, Augsburg : Dr. Erwin Rauner Verlag.

LAMY, Loïc [1995], Le Journal de l'École polytechnique de 1795 à 1831, Sciences et techniques en perspective, 32, 3-96.

Lebesgue, Victor-Amédée [1829a], Extrait d'un mémoire inédit sur les congruences d'un degré quelconque à une seule inconnue, Bulletin du Nord, Journal scientifique et littéraire, 2(1), 23-43.

— [1829b], Extrait d'un mémoire inédit sur les congruences d'un degré quelconque à une seule inconnue, Bulletin du Nord, Journal scientifique et littéraire, 3(1), 255-274.

— [1829c], Extrait d'un mémoire inédit sur les congruences d'un degré quelconque à une seule inconnue, Bulletin du Nord, Journal scientifique et littéraire, 5(1), 19-33.

— [1839], Recherches sur les nombres (suite), Journal de mathématiques pures et appliquées, 4, 9-59.

— [1840a], Sommation de quelques séries, JMPA, I(5), 42-71.

— [1840b], Note sur une formule de Cauchy, JMPA, I(5), 186-188.

LEGENDRE, Adrien-Marie [1827], Recherches sur quelques objets d'analyse indéterminée et particulièrement sur le théorème de Fermat, $M A R S, 6(1)$, $1-60$.

— [1830], Théorie des nombres, Paris : Firmin Didot.

LIBRI, Guglielmo [1825], Analyse indéterminée. Résolution générale de l'équation indéterminée du premier degré à deux inconnues, $A M P A, 16,297-307$.

— [1829], Mémoires de mathématique et de physique, Florence : Ciardetti.

[1831], Mémoire sur quelques formules générales d'analyse, J. Crelle, 7, $57-67$. 
[1832], Mémoire sur la théorie des nombres, J. Crelle, 9, 54-80;169-188; 261-279.

[1835], Mémoires de mathématiques, Berlin : G. Reimer.

Maccioni Ruju, P. Alessandra \& Mostert, Marco [1995], The Life and Times of Guglielmo Libri (1802-1869), Scientist, Patriot, Scholar, Journalist and Thief. A Nineteenth Century Story, Hilversum : Verloren.

Midy, Étienne [1845], Analyse indéterminée du premier degré, $N A M, \mathrm{I}(4)$, $146-152$.

Morel, Thomas [2013], Mathématiques et politique scientifique en Saxe (17651851). Institutions, acteurs et enseignements, Thèse de doctorat, université Bordeaux 1.

PiePer, Herbert [2007], A network of scientific philanthropy : Humboldt's relations with number theorists, dans The Shaping of Arithmetic after C.F. Gauss's Disquisitiones Arithmeticae, édité par C. Goldstein, N. Schappacher \& J. Schwermer, Berlin : Springer, 201-233.

Poinsot, Louis [1845], Réflexions sur les principes fondamentaux de la théorie des nombres, JMPA, I(10), 1-101.

Prouhet, Eugène [1846], Mémoire sur la théorie des résidus dans les progressions géométriques, $N A M, 5,175-187$.

Stern, Moritz Abraham [1834], Démonstration de quelques théorèmes sur les nombres, J. Crelle, 12, 288-291.

Taton, René [1947], Les mathématiques dans le Bulletin de Férussac, Archives internationales d'histoire des sciences, 26, 100-125.

Terquem, Olry [1842], Analyse d'ouvrages nouveaux, NAM, I(1), 49-56.

[1844], Théorie élémentaire des nombres. D'après Euler, Legendre, MM. Gauss et Cauchy, NAM, 3, 204-208; 214-219; 337-344.

Thibault [1843], Théorèmes nouveaux sur les fractions périodiques, NAM, $\mathrm{I}(2), 80-89$.

VERDIER, Norbert [2009a], Le Journal de Liouville et la presse de son temps : une entreprise d'édition et de circulation des mathématiques au $\mathrm{XIX}^{e}$ siècle (1824-1885), Thèse de doctorat, université Paris-Sud.

[2009b], Les journaux de mathématiques dans la première moitié du XIX $^{\mathrm{e}}$ siècle en Europe, Philosophia Scientice, 13(2), 97-126. 
[2012], Panthéons, journaux et salons à Berlin, Londres ou Paris : fabriquer des réseaux de sociabilité savante, dans Panthéons littéraires et savants $\mathrm{XIX}^{e}-\mathrm{XX}^{e}$ siècles, édité par É. Thoizet, N. Wanlin \& G. Weber, Artois : Artois Presses Université, 49-64.

[2013a], Éditer puis vendre des mathématiques avec la maison Bachelier (1812-1864), Revue d'histoire des mathématiques, 19(1), 79-145.

Verhulst, Pierre-François [1827], Théorème de Wilson. Démonstration de M. Verhulst, Correspondance mathématique et physique, 3, 71-72.

Zornow, Anton Robert [1835], De compositione numerorum e cubis integris positivis, J. Crelle, 14, 276-280. 
ANNEXE A* - Nombre d'articles par support éditorial et par décennie

\begin{tabular}{|c|c|c|c|c|c|c|}
\hline Titre & $\begin{array}{l}0 \\
20 \\
\infty \\
\infty \\
-1 \\
1 \\
0 \\
\infty \\
\infty \\
-1\end{array}$ & $\begin{array}{l}0 \\
0 \\
\infty \\
-1 \\
1 \\
-1 \\
0 \\
\infty \\
-1\end{array}$ & 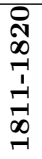 & $\begin{array}{l}\stackrel{0}{\infty} \\
\infty \\
\infty \\
+1 \\
\stackrel{1}{N} \\
\infty \\
\infty \\
-1\end{array}$ & $\begin{array}{l}0 \\
\stackrel{+}{1} \\
\infty \\
-1 \\
1 \\
\stackrel{1}{1} \\
\infty \\
\infty \\
-1\end{array}$ & $\begin{array}{l}0 \\
10 \\
\infty \\
+1 \\
1 \\
-1 \\
+1 \\
\infty \\
-1\end{array}$ \\
\hline $\begin{array}{l}\text { Journal für die reine und angewandte Mathematik } \\
\text { (J.Crelle) }\end{array}$ & 77 & & & 7 & 27 & 43 \\
\hline $\begin{array}{l}\text { Comptes rendus hebdomadaires des séances de l'Acadé- } \\
\text { mie des sciences (CRAS) }\end{array}$ & 31 & & & & 11 & 20 \\
\hline $\begin{array}{l}\text { Livres, dictionnaires, encyclopédies, mémoires publiés } \\
\text { indépendamment }\end{array}$ & 27 & 6 & 3 & 5 & 7 & 6 \\
\hline Journal de mathématiques pures et appliquées (JMPA) & 23 & & & & 8 & 15 \\
\hline Nouvelles annales de mathématiques (NAM) & 17 & & & & & 17 \\
\hline $\begin{array}{l}\text { Bulletin des sciences mathématiques, astronomiques, } \\
\text { physiques et chimiques (Bull. Fér.) }\end{array}$ & 11 & & & 9 & 2 & \\
\hline $\begin{array}{l}\text { Bericht über die zur Bekanntmachung geeigneten } \\
\text { Verhandlungen der Königlich Preußischen Akademie } \\
\text { der Wissenschaften zu Berlin }\end{array}$ & 8 & & & & 3 & 5 \\
\hline $\begin{array}{l}\text { Abhandlungen der Königlichen Preußischen Akademie } \\
\text { der Wissenschaften zu Berlin }\end{array}$ & 7 & & & & 5 & 2 \\
\hline $\begin{array}{l}\text { Mémoires de l'Académie Impériale des Sciences de } \\
\text { Saint-Petersbourg }\end{array}$ & 6 & & & 1 & 3 & 2 \\
\hline Archiv der Mathematik und Physik & 5 & & & & & 5 \\
\hline $\begin{array}{l}\text { Commentationes societatis regiae scientarum } \\
\text { Gottingensis recentiores }\end{array}$ & 5 & 1 & 2 & 1 & 1 & \\
\hline Göttingische Gelehrte Anzeigen & 5 & 1 & 2 & 1 & 1 & \\
\hline $\begin{array}{l}\text { Mémoires de l'Académie des sciences de l'Institut de } \\
\text { France (MARS) }\end{array}$ & 5 & & 1 & 2 & 2 & \\
\hline Exercices d'analyse et de physique mathématique & 3 & & & & & 3 \\
\hline Exercices de mathématiques & 3 & & & 3 & & \\
\hline Journal de l'École polytechnique & 3 & & 2 & & 1 & \\
\hline $\begin{array}{l}\text { Mémoires de l'Académie Royale des Sciences, des } \\
\text { Lettres et des Beaux-Arts de Belgique }\end{array}$ & 2 & & & & & 2 \\
\hline $\begin{array}{l}\text { Mémoires présentés par divers savants à l'Académie des } \\
\text { sciences de l'Institut de France }\end{array}$ & 2 & & & & 1 & 1 \\
\hline Autres** & 14 & 2 & 0 & 6 & 0 & 6 \\
\hline Nombre de périodiques & 31 & 4 & 4 & 13 & 12 & 17 \\
\hline Nombre d'articles & 254 & 10 & 10 & 35 & 72 & 127 \\
\hline
\end{tabular}

*Les zones grisées indiquent les périodes où le support imprimé n'est pas publié.

**Chacune des publications suivantes (Annales de mathématiques pures et appliquées (AMPA), Annali di Fisica, chimica e mathematiche, Milano, Annals of Philosophy, Bulletin de l'Académie royale des sciences, des Lettres et des beaux-arts de Belgique, Bulletin de la classe physico-mathématique de l'Académie impériale des sciences de Saint-Pétersbourg, Bulletin du Nord, Moscou, Bulletin de la Société Philomathique de Paris, Correspondance mathématique et physique, Programm des Gymnasiums zu Torgau (Saxe), Magasin encyclopédique, ou Journal des sciences, des lettres et des arts, Mémoires couronnés par l'Académie royale des sciences et belles-lettres de Bruxelles, Memorie della Reale Accademia delle Scienze in Torino, New series of the mathematical repository, Philosophical Magazine) contient un article pour la période considérée. 
ANNEXE B - Nombre d'articles par auteur et par décennie

\begin{tabular}{|c|c|c|c|c|c|c|c|}
\hline Auteurs & pays & $\begin{array}{l}0 \\
0 \\
\infty \\
\infty \\
1 \\
1 \\
-1 \\
\infty \\
0 \\
-1\end{array}$ & $\begin{array}{l}0 \\
0 \\
\infty \\
\rightarrow 1 \\
\stackrel{1}{1} \\
\infty \\
\infty \\
-1\end{array}$ & $\begin{array}{l}\stackrel{\Omega}{1} \\
\infty \\
\stackrel{1}{1} \\
\stackrel{1}{-1} \\
\infty \\
\rightarrow-1\end{array}$ & $\begin{array}{l}\stackrel{0}{0} \\
\infty \\
\infty \\
\stackrel{1}{1} \\
\stackrel{\sim}{-1} \\
\infty \\
\rightarrow-1\end{array}$ & $\begin{array}{l}\stackrel{0}{+} \\
\infty \\
\stackrel{1}{1} \\
\stackrel{\infty}{\infty} \\
\stackrel{\infty}{-1}\end{array}$ & $\begin{array}{l}0 \\
0 \\
\infty \\
\stackrel{1}{1} \\
\stackrel{1}{+} \\
\infty \\
-1\end{array}$ \\
\hline $\begin{array}{l}\text { Cauchy, Augustin-Louis } \\
(1789-1857)\end{array}$ & France & 37 & 0 & 1 & 6 & 15 & 15 \\
\hline $\begin{array}{l}\text { Dirichlet, Peter Gustav } \\
\text { Lejeune (1805-1859) }\end{array}$ & Allemagne & 22 & & & 6 & 11 & 5 \\
\hline $\begin{array}{l}\text { Eisenstein, Gotthold } \\
(1823-1852)\end{array}$ & Allemagne & 20 & & & & & 20 \\
\hline $\begin{array}{l}\text { Lebesgue, Victor-Amédée } \\
(1791-1875)\end{array}$ & France & 14 & & & 1 & 6 & 7 \\
\hline $\begin{array}{l}\text { Kummer, Ernst Eduard } \\
(1810-1893)\end{array}$ & Allemagne & 13 & & & & & 13 \\
\hline $\begin{array}{l}\text { Gauss, Carl Friedrich } \\
(1777-1855)\end{array}$ & Allemagne & 12 & 4 & 4 & 2 & 2 & \\
\hline $\begin{array}{l}\text { Jacobi, Carl Gustav Jakob } \\
(1804-1851)\end{array}$ & Allemagne & 12 & & & 2 & 7 & 3 \\
\hline $\begin{array}{l}\text { Arndt, Peter Friedrich } \\
(1817-1866)\end{array}$ & Allemagne & 11 & 0 & 0 & 0 & 0 & 11 \\
\hline $\begin{array}{l}\text { Libri, Guglielmo } \\
(1803-1869)\end{array}$ & France & 9 & & 1 & 4 & 4 & 0 \\
\hline $\begin{array}{l}\text { Stern, Moritz Abraham } \\
(1807-1894)\end{array}$ & Allemagne & 8 & & & 1 & 5 & 2 \\
\hline $\begin{array}{l}\text { Terquem, Olry } \\
(1782-1862)\end{array}$ & France & 7 & & & & & 7 \\
\hline $\begin{array}{l}\text { Crelle, August Leopold } \\
(1780-1855)\end{array}$ & Allemagne & 6 & & & & 4 & 2 \\
\hline Poinsot, Louis (1775-1859) & France & 6 & 1 & 2 & 1 & 0 & 2 \\
\hline $\begin{array}{l}\text { Schönemann, Theodor } \\
(1812-1868)\end{array}$ & Allemagne & 6 & & & & 3 & 3 \\
\hline $\begin{array}{l}\text { Bouniakowsky, Victor } \\
(1804-1889)\end{array}$ & Russie & 5 & 0 & 0 & 0 & 2 & 3 \\
\hline $\begin{array}{l}\text { Cournot, Antoine- } \\
\text { Augustin (1801-1877) }\end{array}$ & France & 5 & & & 5 & & \\
\hline $\begin{array}{l}\text { Liouville, Joseph } \\
(1809-1882)\end{array}$ & France & 5 & & & & 1 & 4 \\
\hline $\begin{array}{l}\text { Binet, Jacques Philippe } \\
\text { Marie (1786-1856) }\end{array}$ & France & 4 & 0 & 0 & 0 & 1 & 3 \\
\hline $\begin{array}{l}\text { Legendre, Adrien-Marie } \\
(1752-1833)\end{array}$ & France & 4 & 1 & & 2 & 1 & \\
\hline $\begin{array}{l}\text { Prouhet, Eugène } \\
(1817-1867)\end{array}$ & France & 4 & & & & & 4 \\
\hline $\begin{array}{l}\text { Grünert, Johann August } \\
(1797-1872)\end{array}$ & Allemagne & 3 & & & & 2 & 1 \\
\hline $\begin{array}{l}\text { Schaar, Mathias } \\
(1817-1867)\end{array}$ & Belgique & 3 & & & & & 3 \\
\hline $\begin{array}{l}\text { Galois, Évariste } \\
(1811-1832)\end{array}$ & France & 2 & & & 1 & & 1 \\
\hline
\end{tabular}




\begin{tabular}{|l|l|c|c|c|c|c|c|}
\hline Ivory, James (1765-1842) & Écosse & 2 & 1 & & 1 & & \\
\hline $\begin{array}{l}\text { Kronecker, Leopold } \\
(1823-1891)\end{array}$ & Allemagne & 2 & & & & & 2 \\
\hline Lamé, Gabriel (1795-1870) & France & 2 & & & & & 2 \\
\hline $\begin{array}{l}\text { Ostrogradskij, Mihail V. } \\
(1801-1862)\end{array}$ & Russie & 2 & & & & 2 & \\
\hline $\begin{array}{l}\text { Serret, Joseph-Alfred } \\
(1819-1885)\end{array}$ & France & 2 & & & & & 2 \\
\hline $\begin{array}{l}\text { Autres (auteurs ayant } \\
\text { publié un article) }\end{array}$ & $\begin{array}{l}\text { Allemagne (8) } \\
\text { Angleterre (5) } \\
\text { Belgique (1) }\end{array}$ & 28 & 3 & 2 & 5 & 7 & 11 \\
France (11) & $\begin{array}{l}\text { Italie (2) } \\
\text { Russie (1) }\end{array}$ & & & & & & \\
\hline Nombre d'auteurs & & 56 & 7 & 5 & 16 & 21 & 32 \\
\hline Nombre d'articles & & 254 & 10 & 9 & 31 & 58 & 111 \\
\hline
\end{tabular}

